\title{
Scripta
}

Revista Internacional de Literatura i Cultura Medieval i Moderna

\section{Nota su Lucrezia Borgia nella musica}

\author{
About Lucrezia Borgia in music
}

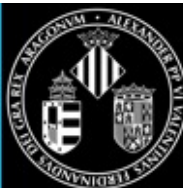

Consuelo Picchi

ielopicchi@gmail.com

Conservatorio di Milano

Riasunto: Nel presente scritto si fa una riflessione sulla presenza di Lucrezia Borgia nella musica ed anche sull'interesse della figlia del papa nella musica del Tromboncino. Ancora, si vede come Donizetti rappresenta Lucrezia nella sua opera.

Parole chiave: Lucrezia Borgia, musica, Tromboncino, Donizetti

\begin{abstract}
In this paper there is a reflection on the presence of Lucrezia Borgia in music and also on the interest of the Pope's daughter in the music of Tromboncino. Still, one sees how Donizetti represents Lucrezia in his work.
\end{abstract}

Keywords: Lucrezia Borgia, music, Tromboncino, Donizetti 
Nei suoi Diari, affascinanti cronache veneziane, scritti tra il XV eil XVI secolo, Marin Sanudo racconta che in occasione delle nozze di Lucrezia Borgia con Alfonso d'Este venne eseguita «una musica del Tromboncino ne la qual si cantò una barzeleta in laude de li sposi: e questo fu inanti principiata la comedia» (Luisi, 1999). Le musiche composte per le sfarzose nozze furono decantate in ogni dove e la fama del compositore, già molto noto presso le più importanti corti italiane, si diffuse con grande clamore.

Tromboncino era giunto a Ferrara nel 1502, chiamato da Alfonso proprio per comporre i brani che avrebbero allietato le feste ferraresi e per festeggiare i nobili sponsali. Per l'occasione, il musicista compose le musiche di scena per l'Asinaria e la Casina di Plauto, oltre che le musiche strumentali di accompagnamento per le danze.

Geneviève Chastenet ci racconta che nulla riusciva a commuovere maggiormente Lucrezia «che ascoltare il celebre Tromboncino cantare le pene d'amore» (Chastenet 1995: 304), spesso accompagnandolo al clavicordo, affidando alla musica l'emozione e l'intensità dei suoi pensieri.

Tromboncino, che la conobbe e la frequentò nella magnificenza del suo palazzo ferrarese, riuscì a incantarla con la grazia e la bellezza delicata delle melodie, la raffinatezza dell'armonia, la suggestione degli strumenti che a gara si univano al canto. E riuscì, tramite la musica, a emozionare il suo animo.

Un animo alla cui affascinante complessità trecento anni dopo un altro grande musicista dedica un'opera straordinaria: Gaetano Donizetti il 26 dicembre 1833 mette in scena alla Scala di Milano Lucrezia Borgia su libretto di Felice Romani, tratto dal dramma Lucrèce Borgia di Victor Hugo e offre un'accorata ricerca umana e psicologica tracciando della donna uno straordinario e potentissimo ritratto.

Nella prefazione, Felice Romani spiega la tematica dell'opera: «Vittore Hugo, dal quale è imitato questo Melodramma, in una Tragedia assai nota [Rigoletto] aveva rappresentato la difformità fisica (son sue parole) santificata dalla paternità: nella Lucrezia Borgia volle significare la difformità morale purificata dalla maternità: il quale scopo, se ben si rifletta, rattempera la nerezza del soggetto, e non fa ributtante la Protagonista». Hugo descrive la figura di Lucrezia come quella di una donna colpevole dei suoi crimini, odiata, temuta e disprezzata, ma pur sempre vittima degli intrighi e delle ambizioni famigliari, che sconvolgono e travolgono la sua esistenza. L'unica possibilità di redenzione e di riscatto spirituale passa attraverso il grande e purissimo amore che nutre per il figlio Gennaro, il quale ignora la vera identità della madre, che scopre soltanto alla fine della tragedia.

Donizetti, nel riprendere il dramma di Hugo, con una musica tra le più alte da lui composte, tratteggia la potente psicologia della protagonista, illuminandone i chiaroscuri caratteriali, la veemente personalità, l'aspirazione possente ad una espiazione esistenziale e coglie l'essenza più profonda della donna, le sue fragilità, la forza potente del suo amore di madre, la disperata violenza con cui difende la vita del figlio.

L'aria Com'è bello!... quale incanto in quel volto onesto e altero! (Prologo, Scena III), che Lucrezia canta osservando il figlio Gennaro addormentato, scolpisce con intensissima apprensione il suo amore 
e nel contempo l' angosciato timore che il figlio possa disprezzarla, una volta conosciuta la verità della sua condizione (Mi risparmia, o ciel, la pena, ch'ei mi debba un dì sprezzar): introdotto dalla dolcezza dell' arpa e accompagnato dalla malinconica morbidezza dei legni, questo brano è uno dei più vibranti scritti da Donizetti, palpitante nel delineare con ricercata introspezione l'immensità del sentimento materno e la sua assoluta abnegazione.

Ancora violenza di sentimenti appare nel duetto con Alfonso (Atto I Scena VI), lo sposo che si crede tradito e ignora che il giovane cui Lucrezia manifesta sì fervido trasporto non è l'amante, bensì il figlio. Lo scambio di accuse e minacce diviene furente, allorché la Borgia si sente perduta, agghiacciata dal terrore che Gennaro venga ucciso dalla brama di vendetta del duca. Oh! A te bada... a te stesso pon mente, di Lucrezia mal cauto marito! (...) Al dolore sottentra la rabbia ... ti potria far la Borgia pentir! Esce tutta la ferocia della madre il cui animo è teso esclusivamente a salvare il bene più grande, pronta ad ogni azione, la più nefasta, la più atroce. Il carattere impetuoso di Lucrezia, il suo vigore, la sua ferrea volontà, è descritto da un accompagnamento sincopato e convulso degli archi, la linea melodica è affannata seppur decisa, l'acuto percussivo esplode sulla parola Borgia, quasi ad enfatizzare il potere distruttivo della nobile donna. Il virtuosismo vocale mira a rappresentare il fervore della passione materna, la frenesia atterrita delle emozioni.

La cupezza del dramma è onnipresente nelle fosche armonie, nei sinistri passaggi in minore delle armonie, nella timbrica orchestrale, resa scura dall'impasto denso dei legni e degli ottoni, dai colpi tenebrosi dei timpani. I lugubri tocchi di campane che accompagnano le voci lontane che si odono durante il banchetto finale (Atto II Scena V) amplificano il terribile detto: La gioia de' profani è un fumo passeggier.

La tensione non cede mai, ogni momento musicale è finalizzato ad analizzare l'atmosfera di orrore, di desolazione che accompagna la protagonista, il suo gravoso percorso esistenziale.

Alla scena conclusiva è affidata tutta la disperazione di Lucrezia accanto al cadavere del figlio (Era desso il figlio mio - Atto II Scena VIII): lo scandaglio psicologico è assoluto, la foga frenetica della madre devastata dal dolore è espressa da un canto dolente, introdotto da un mesto accompagnamento degli archi e da una flebile melodia affidata al malinconico suono dell'oboe. I salti intervallari ascendenti e discendenti lacerano la trama melodica, le note gravi sembrano voler penetrare nel profondo del vuoto che si è creato nella cuore e nella mente di Lucrezia, ormai vaneggiante e fuori di sé, gli acuti squarciano l'ordito melodico e svelano tutta la furia della disperazione. Sul mio capo il cielo avventa il suo strale punitor, la frase che conclude l'opera, è elaborata in un turbinio di vocalizzi, virtuosismi spericolati, trilli, sovracuti.

Esplode così il delirio, l'obnubilamento, l'allucinata sofferenza della madre, che Hugo fa morire per mano del figlio morente e che Donizetti accoglie con immensa pietas, riscattandola attraverso un dolore che redime, purifica, assolve. 


\section{Bibliografia}

Ashbrook, William (1986) Doniretti. La vita, Torino, EDT.

Bellonci, Maria (2003) Lucrezia Borgia, la sua vita e i suoi tempi. Milano, Mondadori.

- (2014) Rinascimento privato, Milano, Mondadori.

Casini, Claudio (1994) Storia della Musica. I: Dall' antichità al Cinquecento, Milano, Rusconi Libri.

Chastenet, Geneviève (1995) Lucręia Borgia. La perfida innocente, Milano, Mondadori.

Della Porta, Dario (ed.) (1983) Dentro Donizetti, Bergamo, Edizioni BOLIS.

Gallico, Claudio (1991) L'età dell'Umanesimo e del Rinascimento, Torino, EDT.

Luisi, Francesco (1999) Tromboncino, Bartolomeo, in DEUMM, vol. VIII, pp. 100-101.

Reese, Gustave (1990) La musica nel Rinascimento, Firenze, Casa Editrice Le Lettere.

Saracino, Egidio (ed.) (1993) Tutti i libretti di Donizetti, Milano, Garzanti.

SCRIPTA, Revista internacional de literatura i cultura medieval i moderna, núm. 9 / juny 2017 / pp. $342-345$ 